\title{
Sequential and co-tunneling behavior in the temperature-dependent thermopower of few-electron quantum $\operatorname{dots}$
}

\author{
R. Scheibner, E.G. Novik, T. Borzenko, M. König, D. \\ Reuter*, A.D. Wieck*, H. Buhmann, and L.W. Molenkamp \\ Physikalisches Institut (EP3), Universität Würzburg, \\ Am Hubland, 97074 Würzburg, Germany and \\ *Lehrstuhl für Angewandte Festkörperphysik, Ruhr-Universität Bochum, \\ Universitätsstraße 150, 44780 Bochum, Germany
}

(Dated: August 10, 2018)

\begin{abstract}
We have studied the temperature dependent thermopower of gate-defined, lateral quantum dots in the Coulomb blockade regime using an electron heating technique. The line shape of the thermopower oscillations depends strongly on the contributing tunneling processes. Between $1.5 \mathrm{~K}$ and $40 \mathrm{mK}$ a crossover from a pure sawtooth- to an intermitted sawtooth-like line shape is observed. The latter is attributed to the increasing dominance of cotunneling processes in the Coulomb blockade regime at low temperatures.
\end{abstract}

Keywords: Thermoelectric and thermomagnetic effects, Coulomb blockade, single electron tunneling

PACS Numbers: 73.50.Lw, 73.23.Hk, 73.63.Kv 
The perspective of scalable semiconductor quantum processing devices feeds an intense interest in quantum dot (QD) structures that contain only a few electrons. 1 For the development of these devices, a detailed knowledge of the underlying electron transport processes is of crucial importance. So far, most of the transport experiments have focused on the electrical conductance $\stackrel{2}{2}$ Although thermoelectrical transport measurements are known to be more sensitive to the details of the electronic structure than conventional transport measurements, $\frac{3}{3}$ little experimental attention has been paid to this kind of measurements on QDs. Within the scope of the Onsager relations, the thermopower $S$, which is given by

$$
S \equiv-\left.\lim _{\Delta T \rightarrow 0} \frac{V_{\mathrm{T}}}{\Delta T}\right|_{I=0}=-\frac{\langle E\rangle}{e T},
$$

relates the average energy $\langle E\rangle$ transfer at a temperature $T$ to the thermovoltage $V_{\mathrm{T}}$ for a given temperature difference $\Delta T$ across the device at zero net current. This additional information about the carrier kinetics is not provided by conventional transport measurements and helps to distinguish between different possible transport regimes. ${ }^{4}$

In the past, thermopower measurements on QDs in the Coulomb blockade $(\mathrm{CB})$ regime have yielded qualitatively different results: either a sawtooth-like line shape, or a line shape similar to the derivative of a Coulomb Blockade (CB) conductance peak is observed when the electrochemical potential is varied in order to change the number of electrons occupying the QD. So far, a sawtooth-like behavior has been observed mainly for many-electron QDs, while derivative-like line shapes are predominantly reported for smaller QDs at low (mK) temperatures: .5 .6 .7

Here, we present thermovoltage measurements on gate defined, lateral QDs, containing a few tens of electrons, which allow us to analyze the low temperature line shape profile in detail. For a series of $\mathrm{CB}$ conductance resonances, the transition is observed from a full sawtooth line shape to a sawtooth that is periodically intermitted by a zero thermovoltage signal while the temperature is lowered from $T=1.5 \mathrm{~K}$ to $T<100 \mathrm{mK}$. This behavior is in qualitative agreement with recent theoretical considerations of Turek and Matveev ${ }^{\underline{8}}$ for many-electron QDs. The transition is associated with an increasing dominance of cotunneling processes for decreasing temperatures. In our measurements we find that the regime of sequential tunneling, which dominates the transport in the vicinity of the $\mathrm{CB}$ resonances, extends much further than anticipated for many-electron dots. This leads to an enhanced 


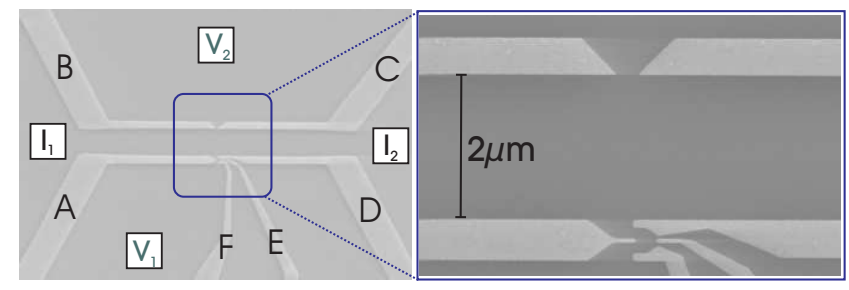

FIG. 1: Scanning electron microscope image of the sample structure. Schottky-gates are labeled $\mathrm{A}, \mathrm{B}, \ldots \mathrm{F}$. Sample areas which serve as current and voltage contacts are labeled $I_{1}, I_{2}$ and $V_{1}, V_{2}$, respectively.

absolute thermopower for few-electron devices.

The measurements are carried out in a top-loading dilution refrigerator at lattice temperatures between $40 \mathrm{mK}$ and 1.5 K. The GaAs/(Al,Ga)As QDs are fabricated by split-gate technology using optical and electron-beam lithography. The two dimensional electron gas (2DEG) is located $70 \mathrm{~nm}$ below the surface, has a carrier density $n_{e}=2.3 \times 10^{15} \mathrm{~m}^{-2}$, and a mobility $\mu=100 \mathrm{~m}^{2} /(\mathrm{Vs})$. The gate structure of the samples is shown in Fig. $\mathbf{1}^{\frac{9}{1}}$ Gates A, $\mathrm{D}, \mathrm{E}$ and $\mathrm{F}$ form the QD with a nominal diameter of approximately $250 \mathrm{~nm}$. The voltage applied to the plunger gate $\mathrm{E}, V_{\mathrm{E}}$, is used to control the number of electrons on the QD. Lowfrequency lock-in techniques are used for the transport experiments. The effective charging energy $E_{\mathrm{C}}^{*}$ is determined From finite bias conductance measurements. In the regime where CB oscillations are observable, $E_{\mathrm{C}}^{*}$ decreases from $3.0 \mathrm{meV}$ to $0.8 \mathrm{meV}$ when the coupling to the reservoirs is increased.

Gates $\mathrm{B}$ and $\mathrm{C}$ define, together with gates $\mathrm{A}$ and $\mathrm{D}$, the electron heating channel, and, at the same time, the reference quantum point contact $\left(\mathrm{QPC}_{\mathrm{ref}}\right)$ in the thermopower experiments. ${ }^{10,11}$ By adjusting the conductance of $\mathrm{QPC}_{\mathrm{ref}}$ to the center of a quantized plateau, its thermopower is approximately zero, $S_{\mathrm{QPC}_{\mathrm{ref}}} \approx 0$. The transverse voltage $V_{\mathrm{T}} \equiv V_{1}-V_{2}$ (c.f. Fig. I) is then directly proportional to the thermopower of the QD. A constant temperature difference across the dot is maintained by passing an ac current of $\nu=13 \mathrm{~Hz}$ through the heating channel. $V_{\mathrm{T}}$ is detected at twice the excitation frequency $(26 \mathrm{~Hz})$. Here, we discuss two QD samples (QD1 and QD2) that have the same gate structure design but adjusted to have a different number of electrons; QD1 (QD2) contains between 15 (30) and 20 (40) electrons. A current of $I_{\mathrm{H}}=9.7 \mathrm{nA}(4.2 \mathrm{nA})$ increases the electron temperature in the heating channel by $\Delta T=9 \mathrm{mK}(3 \mathrm{mK})$ for QD1 (QD2). 

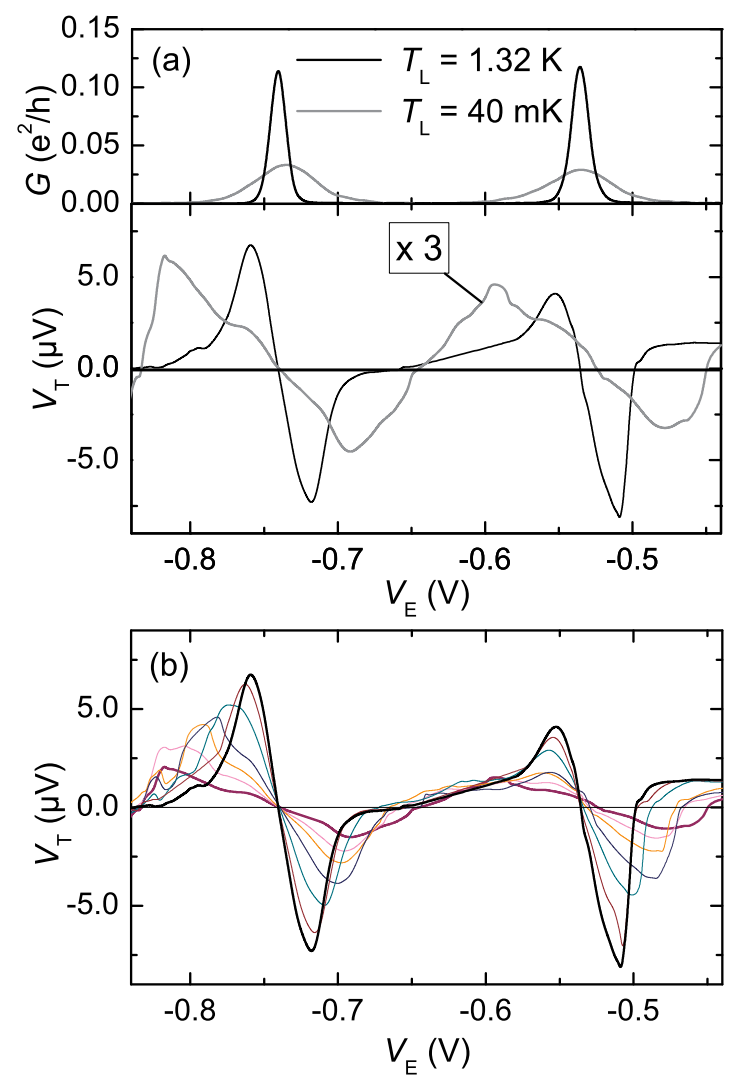

FIG. 2: (color online) (a) Conductance $G$ (upper panel) and the corresponding thermovoltage $V_{\mathrm{T}}$ (lower panel) of $\mathrm{QD} 1$ as a function of the plunger-gate voltage $V_{\mathrm{E}}$. (b) Thermovoltage at seven different temperatures: $T_{\mathrm{L}}=40 \mathrm{mK}$ (black), $66 \mathrm{mK}$ (brown), $158 \mathrm{mK}$ (cyan), $257 \mathrm{mK}$ (blue), 425 $\mathrm{mK}$ (orange), $1.04 \mathrm{~K}$ (pink) and $1.5 \mathrm{~K}$ (purple).

Figure 2 (a) shows the conductance $G$ and the corresponding thermovoltage $V_{\mathrm{T}}$ of QD1 for an electrostatic charging energy $E_{\mathrm{C}}=1.43 \mathrm{meV}$ at lattice temperatures $T_{\mathrm{L}}=40 \mathrm{mK}$ (black lines) and $1.32 \mathrm{~K}$ (grey lines). At $1.32 \mathrm{~K}$, thermal broadening determines the shape of the CB resonances. The corresponding thermovoltage signal shows a sawtooth-like line shape with a maximum in the vicinity of the center of the CB. Additional fine structure on the sawtooth line shape and is due to elevated temperatures and finite level spacings in the QD. At $T_{\mathrm{L}}=40 \mathrm{mK}$ the conduction resonance peaks have an increased height, a reduced width, and are well separated by regimes of (approximately) zero conductance. The line shape of the corresponding thermovoltage now resembles more the negative derivative of the conductance $G$. The values of the thermovoltage extrema increase by a factor of three and 
their positions are shifted towards the CB resonances.

A small asymmetry between the thermovoltage values of positive and negative amplitude is observed for all measurements. Lower tunnel probabilities, for strongly negative plunger gate voltages $V_{\mathrm{E}}$, reduce this asymmetry (c.f. Fig. [2] and Fig. [4). The asymmetry is intrinsic and has been ascribed to multi channel tunneling processes $\frac{12}{2}$ and the energy dependent transmission probability of the tunnel barriers.

Figure 2(b) shows $V_{\mathrm{T}}$ for seven different temperatures in the range from $T_{\mathrm{L}}=1.32 \mathrm{~K}$ down to $40 \mathrm{mK}$. It is evident that the change in line shape occurs continuously. In the vicinity of the $\mathrm{CB}$ resonance the thermovoltage varies linearly with $V_{\mathrm{E}}$, its slope increases with decreasing $T$ while in-between the $\mathrm{CB}$ resonances, a region where $V_{\mathrm{T}} \simeq 0$ develops. The observation of two different line shapes indicates that at different temperatures different transport mechanisms dominate the electronic transport properties.

Near the CB resonances, the charge transport is dominated by sequential tunneling (ST) processes and is explained within the so-called orthodox model $\underline{13}$, where only first order tunneling processes are considered. In-between the resonances, the transport can also be due to ST processes, but only at relatively high temperatures $(\sim 1 \mathrm{~K})$. In ST transport, the average electron energy is proportional to an effective energy gap $E_{\mathrm{g}}$ which is defined as the difference between the Fermi energy of the leads and the energy of the closest QD state. $E_{\mathrm{g}}$ varies linearly between $-E_{C}^{*} / 2$ and $+E_{C}^{*} / 2$ with increasing electrochemical potential of the $\mathrm{QD}, \Phi_{\mathrm{QD}}$, and subsequently jumps back to $-E_{C}^{*} / 2$ at the center of the $\mathrm{CB}$. According to Eq. (II), the thermovoltage follows this sawtooth-like behavior. Thermal smearing at higher temperatures leads to a more sinusoidal variation of $E_{\mathrm{g}}$ and thus the maxima of the thermovoltage oscillations are located slightly away from the center of the CB. The ST mechanism thus explains the line shape at temperatures around $1 \mathrm{~K}$.

We attribute the low $(\mathrm{mK})$ temperature line shape to the occurrence of (inelastic) cotunneling $(\mathrm{CT})$ transport in between the $\mathrm{CB}$ resonances $\underline{\underline{8}}$ At low temperatures, these higher order processes dominate the transport away from the $\mathrm{CB}$ resonance, because the ST processes are thermally activated and thus exponentially suppressed on lowering the temperature, while CT processes scale only according to a power law $\underline{\underline{14}}$ Due to energy conservation, the average energy transferred by cotunneling processes is proportional to the temperature $\left(\left\langle E_{\mathrm{co}}\right\rangle \propto T\right)$. Therefore, the expected thermoelectric signal of CT processes is vanishingly small..$\underline{8}$ Decreasing the sample temperature implies a transition from ST- to CT-dominated 


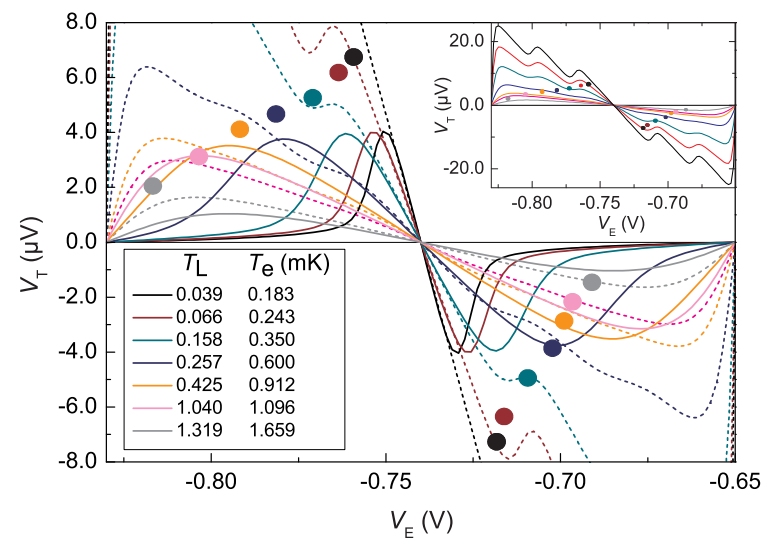

FIG. 3: (color online) Calculated thermovoltage for the orthodox (dotted lines) and CT-included model (full lines) as a function of $V_{\mathrm{E}}$. The dots indicate the maxima of the measured thermovoltage signal. The inset show the orthodox model at full scale.

transport in the $\mathrm{CB}$ regime away from the conductance resonances and thus a suppression of the thermovoltage signal in the corresponding gate voltage ranges. The sawtooth line shape becomes intermitted by regions of (nearly) zero signal amplitude, as observed in Fig. 2.

In order to discuss this transition more quantitatively we compare the thermovoltage resonance at $V_{\mathrm{E}}=-0.73 \mathrm{~V}$ with the behavior of the orthodox (pure $\left.\mathrm{ST}\right)^{13}$ model, and a model that also includes $\mathrm{CT}$ effects ${ }^{8}$. At $T_{\mathrm{L}}=1.32 \mathrm{~K}$ both models exhibit nearly the same thermovoltage amplitude and approximately the same line shape [c.f. Fig. 3]. For this temperature, our QD fulfills the condition $\hbar \Gamma<<k_{B} T<<E_{\mathrm{C}}$, which allows us to extract the relevant model parameters. We obtain $E_{C}^{*}=1.712 \mathrm{meV}, \alpha=\Phi_{\mathrm{QD}} /\left(-e V_{\mathrm{E}}\right)=$ 0.0095, and $G_{l, r}=0.072 e 2 / h$, where $G_{l, r}$ describes the tunnel conductance to the left and right reservoir, respectively. Considering these parameters as temperature independent we calculate the temperature dependent thermovoltage [Fig. 3]. Since the charging energy of the $\mathrm{QD}$ determines the slope of the thermovoltage signal in the close vicinity of the $\mathrm{CB}$ resonances, the direct comparison with the experiment allows us to extract the effective electron temperature $T_{\mathrm{E}}$ under our experimental conditions. The deduced values for $T_{\mathrm{E}}$ are shown in Fig. 3 and have been confirmed independently by fitting the conductance peaks. ${ }^{15.16}$ For clarity, only the maxima of the measured thermovoltage are indicated in the 
figure, together with the results of the model calculations.

In the orthodox model, a sawtooth line shape is predicted for all temperatures (Fig. 3). The wiggles at the declining slope of the sawtooth come from excited states and have the periodicity of the level spacing. At the same time, the CT-included model does indeed reproduce a transition from a sawtooth to a periodically suppressed sawtooth line shape. However, while the CT-included model predicts an approximately constant peak amplitude, the experiments show a strong increase in peak amplitude with decreasing temperature. In addition, the model does not predict the gate voltage position of the maxima correctly. A comparison of Fig. 2 and Fig. 3 reveals that in the experiment the linear increase of the thermovoltage around the CB resonance extends much further than anticipated by the CT-included model, and rather follows the behavior of the orthodox model i.e., the voltage range where ST dominates the transport is larger than given by the CT-included model. In other words, the CT-included model overestimates the influence of CT processes for our few-electron QD. The reason is that the CT-included model assumes a negligible energy spacing of the QD states $\left(\delta E_{\mathrm{QD}}<<k_{B} T\right)$, as applicable for metallic dots. For the present few-electron $\mathrm{QD} \delta E_{\mathrm{QD}}$ clearly is not negligible, the energy gap between the ground and the first excited state is of the order of $250 \mu \mathrm{eV}$. Even at temperatures around $1 \mathrm{~K}$, only a few excited states are available for transport, which reduces the probability for co-tunnel events.

For comparison, we measured a second QD sample, which has the same layout as QD1 (c.f. Fig. 1). QD2 exhibits a similar charging energy $\left(E_{\mathrm{C}}^{*} \approx 1.5 \mathrm{meV}\right)$ but smaller level spacing $(\delta E \sim 50 \mu \mathrm{eV})$ presumably due to variations in the potential landscape in the 2DEG. ${ }^{17}$ Figure 4 (a) shows the change of the thermovoltage line shape for a series of CB resonances. Clearly, this sample is further into the many electron limit. The change in line shape with temperature is still present. It becomes less pronounced for more positive gate voltages, where the coupling of the QD states to the leads increases. The line shape close to the $\mathrm{CB}$ resonance at $V_{\mathrm{E}}=-1.9 \mathrm{~V}$ has been used to evaluate the model calculations similar to QD1. Fig. 4 (b) confirms confirms that again the CT-included model overestimates the influence of CT processes comparable to the case of QD1.

In summary, the thermopower of few-electron QDs reveals a transition of the line shape as a function of the QD potential from a full sawtooth to an intermitted sawtooth behavior. This transition is directly related to a change in the dominant transport processes. While close to the $\mathrm{CB}$ resonances, sequential tunneling processes dominate, the full $\mathrm{CB}$ regime 

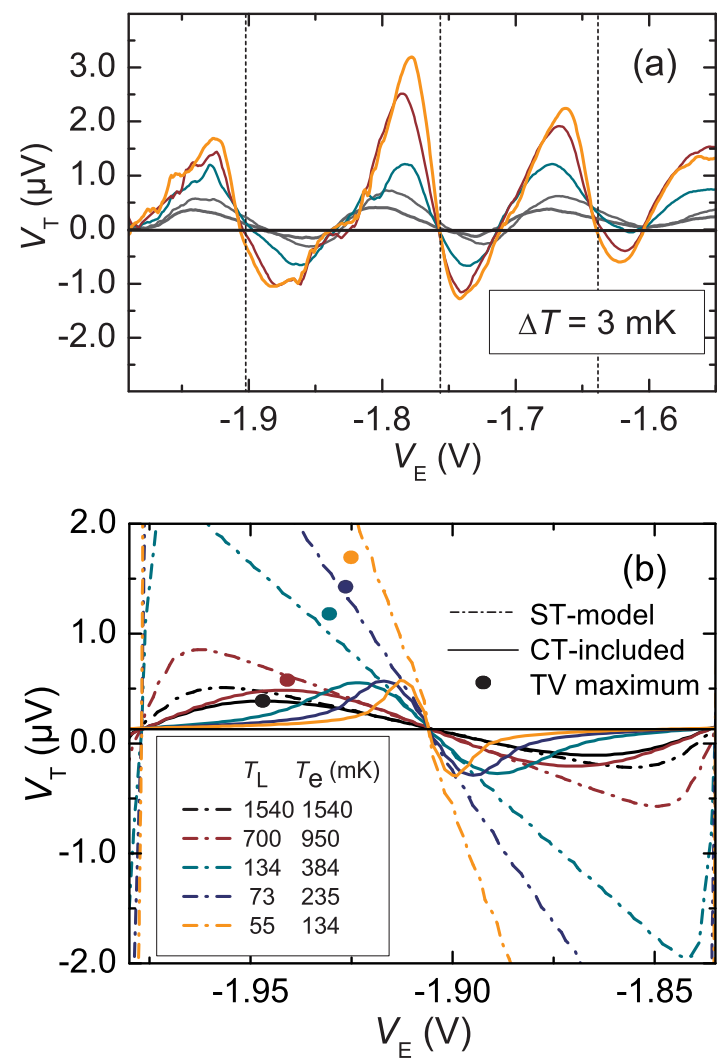

FIG. 4: (color online) (a) Thermovoltage for a series of CB resonances as a function of the plunger gate voltage $V_{\mathrm{E}}$ for $\mathrm{QD} 2$. The positions of the $\mathrm{CB}$ resonances are indicated by vertical dashed lines. (b) Calculated thermovoltage for the orthodox (dash-dotted lines) and CT-included model (full lines) as a function of $V_{\mathrm{E}}$. The dots indicate the maxima of the measured thermovoltage signal near the resonance at $V_{\mathrm{E}}=-1.9 \mathrm{~V}$.

is dominated by inelastic cotunneling processes. Compared with many-electron (metallic) QDs, the regime of sequential tunneling is extended to a wider gate voltage range for fewelectron QDs, which results in an increasing thermopower peak amplitude with decreasing temperature.

We wish to thank C. Gould and J. Weis for valuable discussions. We gratefully acknowledge the financial support of the DFG (Mo771/5-2) and the Office of Naval Research 
(04PR03936-00).

1 D. Loss and D.P. DiVincenzo, Phys. Rev. A 57, 120 (1998).

2 L.P. Kouwenhoven, C.M. Marcus, P.L. McEuen, S. Tarucha, R.M. Westervelt, and N.S. Wingreen, Mesoscopic Electron Transport (Kluver Academic, Dordrecht, 1997), p. 105-214.

3 J.M. Ziman, Electrons and Phonons, Oxford University Press (1960).

4 R. Scheibner, H. Buhmann, D. Reuter, M. N. Kiselev, and L. W. Molenkamp Phys. Rev. Lett., 95, $176602(2005)$.

5 A.A.M. Staring, L.W. Molenkamp, B.W. Alphenaar, H. van Houten, O.J.A. Buyk, M.A.A. Mabesoone, C.W.J. Beenakker, and C.T. Foxon, Europhys. Lett., 22, 57 (1993).

6 S. Möller, H. Buhmann, S.F. Godijn and L.W. Molenkamp, Phys. Rev. Lett. 81, 5197 (1998); S.F. Godijn, S. Möller, H. Buhmann, L.W. Molenkamp, and S.A. vanLangen, Phys. Rev. Lett. 82, 2927 (1999).

7 A.S. Dzurak, C.G. Smith, C.H.W. Barnes, M. Pepper, L. Martin-Moreno, C.T. Liang, D.A. Ritchie, and G.A.C. Jones, Phys. Rev. B 55, R10197 (1997).

8 M. Turek and K.A. Matveev, Phys. Rev. B 65, 115332 (2002).

9 M. Ciorga, A. S. Sachrajda, P. Hawrylak, C. Gould, P. Zawadzki,S. Jullian, Y. Feng, and Z. Wasilewski, Phys. Rev. B 61, R16315 (2000).

10 B. L. Gallagher, T. Galloway, P. Beton, J.P. Oxley, S.P. Beaumont, S. Thoms, and C.D.W. Wilkinson Phys. Rev. Lett. 64, 2058 (1990); L.W. Molenkamp, Th. Gravier, H. van Houten, O.J.A. Buijk, M.A.A. Mabesoone, and C.T. Foxon Phys. Rev. Lett. 68, 3765 (1992).

11 H. van Houten, L.W. Molenkamp, C.W.J. Beenakker, and C.T. Foxon, Semicond. Sci. Technol., 7, B215 (1992); P. Streda, J. Phys. Condens. Matter 1, 1025 (1989); L.W. Molenkamp, H. van Houten, C.W.J. Beenakker, R. Eppenga, and C.T. Foxon, Phys. Rev. Lett. 65, 1052 (1990).

12 S.-J. Xiong, and Y. Yin, Phys. Rev. B 66, 153315 (2002); S.-J. Xiong, and Y. Xiong, Phys. Rev. Lett. 83, 1407 (1999).

13 C.W.J. Beenakker and A.A.M. Staring, Phys. Rev. B 46, 9667 (1992).

14 D.V. Averin, and Yu. V. Nazarov, Phys. Rev. Lett. 65, 2446 (1990).

15 C.W.J. Beenakker, Phys. Rev. B 44, 1646 (1991).

16 S. Vorojtsov, Int. Journal of Modern Physics B, 18, 3915 (2004). 
17 J.J. Koonen, H. Buhmann, and L.W. Molenkamp, Phys. Rev. Lett. 84, 2473 (2000). 